\title{
Terahertz Applications of Frequency Selective Surfaces: Analysis, Design, Fabrication and Testing
}

\author{
J. A. Bossard*, L. Li, J. A. Smith, D. H. Werner, T. S. Mayer, R. P. Drupp, and Y. Tang \\ The Pennsylvania State University, Department of Electrical Engineering \\ jab678@psu.edu and dhw@psu.edu
}

\section{Introduction}

Frequency Selective Surfaces (FSS) are well known in the literature for their filtering characteristics at microwave and millimeter wave frequencies [1]. Traditional FSS filters are comprised of a doubly periodic metallic screen printed on a dielectric substrate and are sometimes referred to in the infrared (IR) as metallodielectric photonic crystals (MDPCs). Metallodielectric FSS have also been synthesized at microwave frequencies to exhibit negative refractive index and zero refractive index behavior [2]. This research naturally extends to synthesizing negative index metamaterials (NIM) and zero index metamaterials (ZIM) in the IR. For applications at visible wavelengths, metallic losses degrade the performance of metallodielectric FSS, whereas low loss dielectric materials are readily available. Thus, all-dielectric FSS (DFSS) are also being investigated in this work for filtering applications in the near-IR and optical regimes. In this paper, we report on the successful design and experimental verification of single layer IR FSS with excellent multiband filter responses synthesized using fractal and genetic algorithm (GA) techniques $[3,4]$. Additional rules were incorporated into our GA synthesis algorithm to improve the fabrication accuracy of the resulting IR FSS designs [5]. The synthesis of DFSS using a GA is also discussed along with a design example. Finally, some results of FSS NIMs designed for low loss operation in the IR will be presented.

\section{Synthesis of Metallodielectric IR FSS}

A full-wave periodic method of moments (PMM) analysis technique was employed in this work to perform the electromagnetic simulations of metallodielectric FSS [1]. Published metallic loss models were incorporated in the PMM simulation. Optical contact lithography was chosen as the method for fabricating the metallodielectric IR FSS. The metallic screen is $75 \mathrm{~nm}$ thick aluminum deposited on a $0.5 \mu \mathrm{m}$ or $4 \mu \mathrm{m}$ polyimide substrate. Transmission measurements were performed using Fourier transform infrared (FTIR) spectroscopy.

FSS with fractal metallic screen geometries have been investigated at IR frequencies for achieving multiple resonances from a single layer screen design [3]. Figure 1 shows an array of fractal cross dipole patches with the primary and secondary cross dipoles highlighted. The primary and secondary cross dipoles with arm lengths of 17 and $7 \mu \mathrm{m}$, respectively, and a linewidth of $1.5 \mu \mathrm{m}$ are expected to each give rise to a stopband at their corresponding resonant wavelengths. The dimensions were chosen such that they would exhibit a dual-band response in the far-IR spectrum and so they could be readily fabricated using optical contact lithography. The spacing between the fractal elements was optimized via simulation for a strong secondary stopband and found to yield the best performance at $1.2 \mu \mathrm{m}$. Figure 1 also shows a scanning electron microscope (SEM) image of the fabricated structure. The measured response shown in Figure 2 reveals two strong stopbands in the transmission spectrum at $6.9 \mathrm{THz}$ and $20.3 \mathrm{THz}$ and excellent agreement between measurement and simulation. Design of metallodielectric FSS using fractal screen geometries has limitations, such as the relative positioning of bands when using cross-dipole fractal FSS screen elements. To overcome these constraints, we consider a much more general design synthesis technique based on a robust Genetic Algorithm (GA) optimization procedure [4]. 
Because the substrate for each FSS design was chosen a priori, the only parameters optimized by the GA were the metallic screen geometry and the unit cell size. Eight-fold symmetry was applied to the unit cell geometry to achieve polarization independence. In order to simplify fabrication, a set of constraints based on fabrication rules were developed and incorporated into the GA [5]. The fitness function given in (1) minimizes the reflectance, $R$, at passband frequencies and the transmittance, $T$, at stopband frequencies.

$$
\text { Fitness }=\sum_{\text {pass }} R+3 \sum_{\text {stop }} T
$$

A GA synthesized IR FSS design with a unit cell size of $36.9 \mu \mathrm{m}$ on a side is shown in Figure 3. This design was optimized on a $0.5 \mu \mathrm{m}$ polyimide substrate for two stopbands at $3.8 \mathrm{THz}$ and $7 \mathrm{THz}$ and passbands elsewhere over the range from $1 \mathrm{THz}$ to $10 \mathrm{THz}$. A population size of 75 was evolved over 300 generations, and the metallic loss of aluminum at $10 \mathrm{THz}$ was included in the fitness evaluation. The optical microscope image of the fabricated structure reveals an accurately fabricated screen geometry. The simulated and measured transmission spectra for this design are also shown in Figure 3.

A second GA synthesized IR FSS design was optimized on a $4 \mu \mathrm{m}$ polyimide substrate to have two stopbands at $2.7 \mathrm{THz}$ and $5.9 \mathrm{THz}$ and passbands elsewhere over the range from $1 \mathrm{THz}$ to $10 \mathrm{THz}$. The optimized unit cell is shown in Figure 4 along with an optical microscope image of the fabricated structure. The unit cell dimension is $35.9 \mu \mathrm{m}$ on a side. The simulated and measured transmission spectra show excellent agreement between the optimization goals and the response of the fabricated structure.

\section{Synthesis of All-Dielectric IR FSS}

Inhomogeneous all-dielectric periodic structures that act as frequency selective filters have been investigated over the years. At millimeter frequencies or higher, such dielectric arrays offer the advantage of low absorption loss as compared to metallic screens. A typical all-dielectric frequency selective surface (DFSS) consists of two or more different materials distributed periodically throughout the layer. In our work, a Finite ElementBoundary Integral (FE-BI) method is used to efficiently model the material inhomogeneities and periodic boundaries required for the analysis of DFSS. Another important advantage of the FE-BI method is that it can be used in conjunction with a GA to provide a powerful tool for the design optimization of DFSS for specific low-loss applications, including filters, radomes, and even Negative Index Materials (NIM) [6].

The specific structure considered here to illustrate the GA design process for DFSS consists of a thin dielectric slab made up of two different materials. The unit cell of the DFSS is volumetrically pixelized into $16 \times 16$ small cubes, and the GA is used to optimize the distribution of the two materials for a desired frequency response. The dielectric constant of the two materials, the periodicity of the DFSS, and the thickness of the dielectric layer is chosen a priori. In order to get a polarization insensitive frequency response from the DFSS, a rotationally symmetric scheme is enforced on the material distribution, which means that only a quarter of the unit cell needs to be optimized.

$$
\text { Fitness }=2\left\{\sum_{\substack{\text { Stop-band(s) } \\ \text { transmission }>0.1}}(\text { transmission }-0.1)^{3 / 2}+\sum_{\substack{\text { Pass-band(s) } \\ \text { reflection }>0.2}}(\text { reflection }-0.2)^{3 / 2}\right\}
$$

The goal of the GA in this example is to provide a single stopband at $90 \mathrm{THz}$. The fitness function of the GA, which is given in (2), was designed to simultaneously minimize the 
transmission coefficient in the stopband and reflection coefficient in the passband. The configuration of the DFSS unit cell as well as the corresponding scattering responses of the periodic structure are shown in Figure 5. Note that the scattering responses of the DFSS are the same for both TE and TM polarizations. The periodicity of the DFSS is $2.54 \mu \mathrm{m}$, and the thickness of the DFSS is $1.08 \mu \mathrm{m}$. The relative dielectric constants of the two materials are 1.82 and 2.82 respectively.

\section{Conclusions}

Metallodielectric IR FSS filters were synthesized using fractal and GA techniques with fabrication constraints. Measured results show excellent agreement with PMM simulation. The synthesis of DFSS for near-IR filter applications was performed using a GA. The advantages of DFSS include low loss at visible wavelengths and polarization independent filter responses. Designs for low loss IR NIMs based on FSS were also discussed. Future work includes fabricating FSS and DFSS filters that operate at near-IR and visible wavelengths.

Acknowledgements: This research was supported by the NSF MRSEC, Center for Nanoscale Science, Grant No. DMR-0213623.

\section{References}

[1] B. A. Munk, Frequency Selective Surfaces: Theory and Design. New York: Wiley, 2000.

[2] M. A. Gingrich, and D. H. Werner, "Synthesis of low / zero index of refraction metamaterials from frequency selective surfaces using genetic algorithms," IEE Electronics Letters, Vol. 41, No. 23, Nov. 10, 2005.

[3] R. P. Drupp, J. A. Bossard, Y-H. Ye, D. H. Werner, and T. S. Mayer, "Dual-band infrared metallodielectric photonic crystals," Appl. Phys. Lett., Vol. 85, pp. 18351837, Sept. 2004.

[4] R. P. Drupp, J. A. Bossard, D. H. Werner, and T. S. Mayer, "Single-layer multi-band infrared metallodielectric photonic crystals designed by genetic algorithm optimization," Appl. Phys. Lett., Vol. 86, pp. 081102-1-081102-3, Feb. 2005.

[5] J. A. Bossard, J. A. Smith, D. H. Werner, T. S. Mayer, and R. P. Drupp, "Multiband planar infrared metallodielectric photonic crystals designed using genetic algorithms with fabrication constraints", IEEE AP-S Symposium, Washington, DC, Vol. 1A, pp. 705-708, July 3-8, 2005.

[6] Ling Li, and D. H. Werner, "Design of all-dielectric frequency selective surfaces using genetic algorithms combined with the finite element-boundary integral method", IEEE AP-S Symposium, Washington, DC, Vol. 4A, pp. 376-379, July 3-8, 2005 .
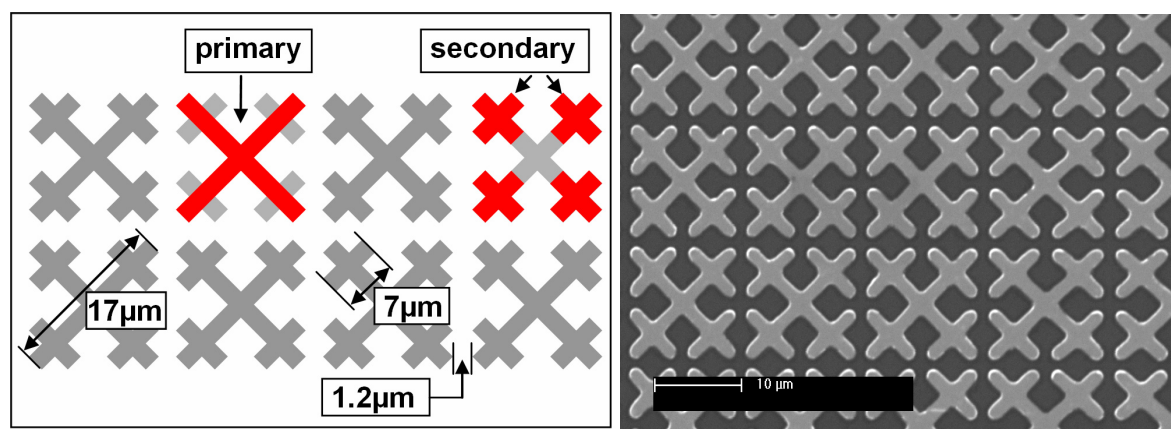

Figure 1. Fractal cross dipole IR FSS illustration with design parameters (left) and SEM image of fabricated structure (right). 


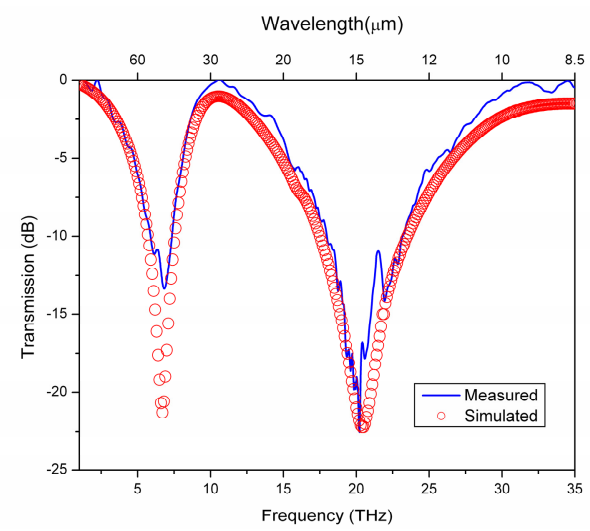

Figure 2. Simulated and measured transmission spectra of the fractal cross dipole IR FSS on $0.5 \mu \mathrm{m}$ polyimide substrate shown in Figure 1.
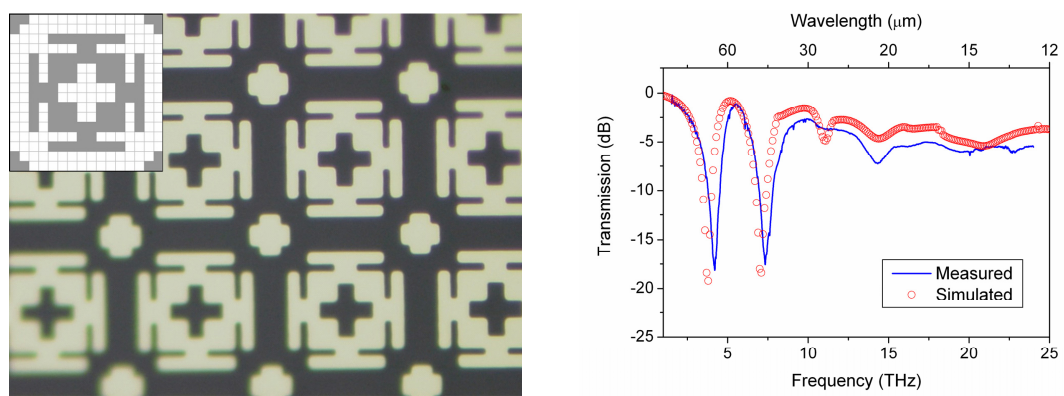

Figure 3. Optical microscope image of an IR FSS synthesized via GA on a $0.5 \mu \mathrm{m}$ polyimide substrate for stopbands at $3.8 \mathrm{THz}$ and $7 \mathrm{THz}$ with inset unit cell geometry (left). Simulated and measured transmission spectra (right).
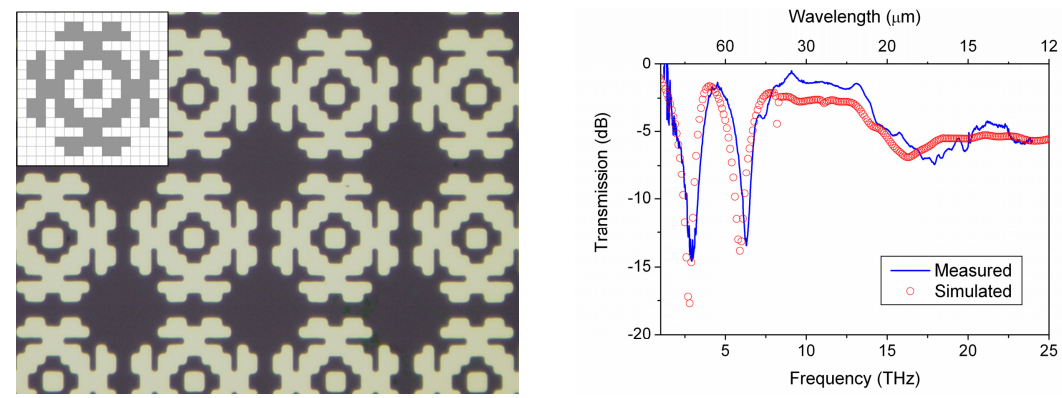

Figure 4. Optical microscope image of a GA synthesized IR FSS on a $4 \mu \mathrm{m}$ polyimide substrate with stopbands at $2.7 \mathrm{THz}$ and $5.9 \mathrm{THz}$ with inset unit cell geometry (left). Simulated and measured transmission spectra (right).
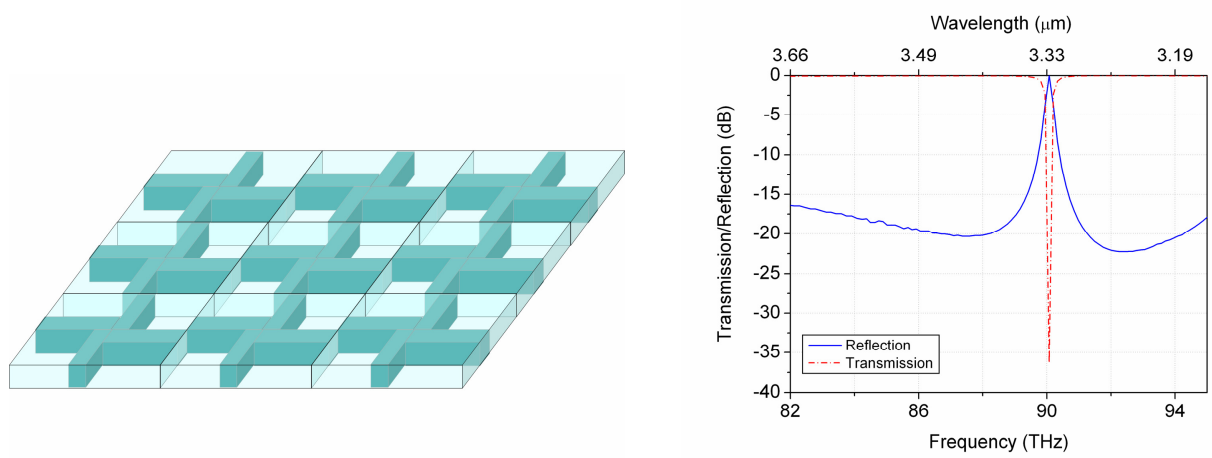

Figure 5. Geometry of GA synthesized DFSS polarization independent filter with a stopband at $90 \mathrm{THz}$ (left) and simulated scattering response (right). 